Nervenarzt 2019 $\cdot 90: 99-106$

https://doi.org/10.1007/s00115-018-0670-6

(c) Der/die Autor(en) 2019

CrossMark

\author{
Markus M. Nöthen ${ }^{1}$ Franziska Degenhardt ${ }^{1}$ Andreas J. Forstner ${ }^{1,2}$ \\ ${ }^{1}$ Institut für Humangenetik, Universitätsklinikum Bonn, Bonn, Deutschland \\ ${ }^{2}$ Zentrum für Humangenetik, Philipps-Universität Marburg, Marburg, Deutschland
}

\title{
Durchbrüche im Verständnis der molekularen Ursachen psychiatrischer Störungen
}

affektiven Störungen zusammen. Wegen ihrer Häufigkeit und Schwere sind diese Krankheiten von den psychiatrischen Erkrankungen des Erwachsenenalters am intensivsten untersucht. Wir geben im Artikel auch einen Ausblick auf Möglichkeiten der Translation genetischer Erkenntnisse in Diagnostik und Therapie sowie auf Strategien zur weiteren Aufklärung der krankheitsrelevanten biologischen Mechanismen.

\section{Genidentifizierung bei psychiatrischen Erkrankungen} Unterfangen mit einer Vielzahl nicht replizierbarer Befunde. Aus heutiger Sicht litten die damaligen Studien entweder an zu geringer Power (Überschätzung der zu erwartenden Effektstärken häufiger genetischer Varianten), mangelnder Abdeckung des Genoms (Untersuchung weniger Varianten in einzelnen Kandidatengenen) oder aber an einer unkritischen Anwendung von Methoden, die bei der Analyse monogener Krankheiten außerordentlich erfolgreich waren (Kopplungsuntersuchung mit anschließender Positionsklonierung), bei psychiatrischen Krankheiten aber nicht der Komplexität des genetischen Beitrags Rechnung trugen.

Mittlerweile hat sich die Situation grundlegend geändert. Für viele psychiatrische Erkrankungen sind krankheitsrelevante Regionen im menschlichen Genom zweifelsfrei identifiziert worden. Die genetischen Befunde bedeuten einen wichtigen Schritt zum Verständnis der molekularen Ursachen. In unserem Artikel fassen wir den Stand der Kenntnis für die Gruppe der schizophrenen und
Auf die erstmalige Sequenzierung des menschlichen Genoms im Rahmen des Humangenomprojekts [28] folgte seit Beginn der 2000er-Jahre die systematische Charakterisierung der individuellen Ausprägungen des menschlichen Genoms. Damit einher ging ein rasanter technologischer Fortschritt zur effizienten Darstellung der genomischen Variabilität. Mit der Einführung von Mikroarrays, die genomweit häufige genetische Varianten (Einzelnukleotidpolymorphismen, „single nucleotide polymorphisms" [SNPs]) analysierten (sog. SNP-Arrays), gelang seit Mitte der 2000er-Jahre der Durchbruch in der Analyse multifaktorieller Krankheiten durch die Anwendung genomweiter Assoziationsstudien (GWAS). Auch die genomweite Erfassung seltener Varianten profitierte von der Mikroarray-Entwicklung. Durch eine tiefergehende Analyse der SNP-Array-Intensitätsdaten sowie mit speziell zu diesem Zweck entwickelten Array-Technologien konnten genomweit Verluste oder Zugewinne genetischen
Materials (Deletionen/Duplikationen, Kopienzahlvarianten [„copy number variants“, CNVs]) identifiziert werden. Die Mikroarray-Technologien erlauben die Untersuchung von DNA-Proben im Hochdurchsatz und zu stetig sinkenden Kosten.

\section{1) Mit Bildung internationaler Genomkonsortien gelang der entscheidende Durchbruch}

Der entscheidende Durchbruch für die psychiatrischen Erkrankungen gelang letztendlich mit der Bildung internationaler Konsortien (z.B. Psychiatric Genomics Consortium, PGC, [55]), in denen die Datensätze der einzelnen Arbeitsgruppen zusammengeführt und gemeinsam mit neuesten statistischen Verfahren analysiert wurden. Dahinter stand die Einsicht, dass sowohl bei der Analyse häufiger Varianten - wegen der jeweils kleinen Effektstärken - als auch bei der Analyse seltener Varianten u. a. durch die Vielzahl möglicherweise mutierter Gene - die Stichprobengröße kritisch für den Erfolg ist.

\section{Befunde bei schizophrenen Störungen}

Schizophrenie (SCZ) hat eine geschätzte Gesamterblichkeit (Heritabilität) von etwa $60-80 \%[4,40]$. Die bisher durchgeführten GWAS konnten mehr als 140 krankheitsassoziierte Loci identifizieren $[39,49]$. Unter Einbeziehung der nichtsignifikanten Varianten erklären die unter- 
Tab. 1 Kopienzahlvarianten mit substanziellem Beitrag zur Ätiologie der Schizophrenie. (Aus [32])

\begin{tabular}{|c|c|c|}
\hline CNV & Odds Ratio & Häufigkeit in Patienten \\
\hline del 1q21.1/dup 1q21.1 & 4 & $2,8 / 1000$ \\
\hline del $2 \mathrm{p} 16.3$ & 15 & $1,7 / 1000$ \\
\hline del 3q29 & a & $0,8 / 1000$ \\
\hline dup $7 q 11.23$ & 15 & $0,8 / 1000$ \\
\hline del $15 q 13.3$ & 15 & $1,3 / 1000$ \\
\hline del 16p11.2, distal & 20 & $0,5 / 1000$ \\
\hline dup 16p11.2, proximal & 10 & $3,3 / 1000$ \\
\hline del $22 q 11.2$ & $>65$ & $3,0 / 1000$ \\
\hline \multicolumn{3}{|c|}{$\begin{array}{l}\text { CNV "Copy number variants", del Deletion, dup Duplikation } \\
\text { aDeletionen in 3q29 wurden bisher nicht in Kontrollen nachgewiesen; daher ist eine exakte Berech- } \\
\text { nung des Odds Ratios nicht möglich }\end{array}$} \\
\hline
\end{tabular}

suchten SNPs eine Heritabilität von etwa 20-30\% [39].

In der bisher größten GWAS des PGCs wurden die Genotypdaten von etwa 37.000 Patienten mit SCZ und 113.000 Kontrollprobanden analysiert und insgesamt 108 genomweit signifikante Loci identifiziert [49]. Die assoziierten Loci implizieren u.a. Mechanismen der Kalziumsignalübertragung, der Signalübertragung an glutamatergen Synapsen und Prozesse des Immunsystems [49]. Das stärkste Assoziationssignal ist in der Major-histocompatibility-complex(MHC)-Region auf Chromosom 6 lokalisiert, welche relevante Gene für die erworbene Immunität enthält [49]. Dieses Signal kann - zumindest partiell durch strukturelle Variationen in einem Gen, welches für den Komplementfaktor C4 kodiert, erklärt werden. Die unterschiedlichen C4-Allele haben offenbar (über Zwischenschritte) einen Einfluss auf die während der Entwicklung des Gehirns stattfindende Eliminierung von Synapsen („synaptic pruning“; [51]).

\section{》) Neumutationen verschieben das allelische Spektrum Richtung seltene Varianten}

Neben häufigen sind auch seltene genetische Varianten mit der SCZ in Zusammenhang gebracht worden. Ein relevanter Beitrag seltener Varianten war aufgrund der verminderten durchschnittlichen Reproduktionsrate erkrankter Personen bereits vermutet worden [41]. Die trotz verminderter Reprodukti- onsrate gleichbleibende Häufigkeit in der Bevölkerung ließe sich zumindest teilweise durch einen Beitrag von Neumutationen erklären. Ein größerer Anteil von Neumutationen an den genetischen Ursachen einer Erkrankung führt zur Verschiebung des allelischen Spektrums Richtung seltene Varianten.

Zunächst gelang der Nachweis eines Beitrags seltener Varianten für CNVs. In der weltweit größten CNV-Analyse zu psychiatrischen Erkrankungen wurden die Daten von ca. 21.000 Patienten mit SCZ und 20.000 Kontrollprobanden analysiert. CNVs in acht chromosomalen Loci waren genomweit signifikant mit SCZ assoziiert (• Tab. 1). Die identifizierten pathogenen Deletionen und Duplikationen haben moderate bis relativ große Effektstärken (Odds Ratio ca. 4-65). Ins$1 \%$ der Patienten mit SCZ nachweisbar [32].

Zum systematischen Nachweis seltener nur ein oder wenige Nukleotid(e) umfassender Varianten werden heutzutage typischerweise Sequenzierungen der gesamten kodierenden Sequenzen des Genoms durchgeführt (sog. Exomsequenzierungen). Insgesamt ist die Anzahl an exomweiten Sequenzierungsstudien bei Patienten mit SCZ noch relativ überschaubar (u.a. $[17,46]$ ); bisher wurde nur für ein Gen - SETD1A auf Chromosom 16 - eine genomweit signifikante Assoziation zwischen seltenen pathogenen Varianten und SCZ berichtet [52].

In der bisher größten durchgeführten exomweiten Sequenzierungsstudie zum gesamt sind pathogene CNVs bei etwa direkten Nachweis von Neumutationen [17] wurde bei der Untersuchung von knapp 620 Trios (Patient plus Eltern) zwar keine allgemein erhöhte Neumutationsrate gefunden, die detaillierte Analyse zeigte jedoch, dass Neumutationen in spezifischen Gengruppen statistisch signifikant angereichert waren. $\mathrm{Zu}$ diesen gehörten Gene, in denen zuvor pathogene Neumutationen bei Patienten mit Intelligenzminderung oder Autismusspektrumstörung berichtet worden waren, z. B. Zielgene des „fragile x mental retardation protein" (FMRP) und ausgewählte Gene (ARC/NMDAR) der „postsynaptic density“ (PSD).

\section{Befunde bei affektiven Störungen}

Die Heritabilität bei der majoren Depression („major depression“, MD) wird auf etwa $40 \%$ [35] und bei der bipolar affektiven Störung („bipolar disorder“, BD) auf etwa $60-85 \%$ geschätzt $[4,30]$.

Mit GWAS konnten mittlerweile eine größere Zahl krankheitsassoziierter genomischer Loci sowohl für BD als auch für MD identifiziert werden (u. a. $[9,34$, 47]). Die aktuell größten Untersuchungen von GWAS-Datensätzen wurden bei affektiven Störungen im Rahmen von Metaanalysen des PGC durchgeführt.

Bei BD konnten mit Genotypdaten von etwa 30.000 Patienten und 170.000 Kontrollpersonen 30 genomweit signifikante Loci identifiziert werden [54]. Bei MD wurden größere Stichproben benötigt, was man sich nicht nur mit der geringeren Heritabilität, sondern vor allem mit der im Vergleich zu BD größeren Heterogenität erklärt. In der 2018 erschienen Metaanalyse des PGC führte die Untersuchung von etwa 135.000 MD-Patienten und 345.000 Kontrollpersonen zur Identifizierung von 44 genomweit signifikanten Loci [61]. Bezieht man die Informationen aller SNPs mit ein, erklären die untersuchten Varianten bei BD eine Heritabilität von etwa $17-23 \%$ und bei MD von etwa $9 \%[54,61]$.

Die assoziierten Loci und die durch sie implizierten Gene zeigen eine Häufung in bestimmten Stoffwechselwegen. Für BD gibt es Hinweise auf Mechanismen der Histonmodifikation, der Kalzi- 
Hier steht eine Anzeige.

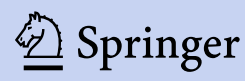


umsignalübertragung und der Endocannabinoidsignalwege $[37,45,54]$. Bei MD zeigte sich unter anderem eine Anreicherung in Genen, die bei der Organisation von Zellkontakten, der synaptischen Übertragung und an Kalziumsignalwegen beteiligt sind $[37,61]$.

\section{) Mit MD und BD assoziierte Loci zeigen eine Häufung in bestimmten Stoffwechselwegen}

Kopienzahlvarianten scheinen bei affektiven Störungen im Vergleich zur SCZ eine geringere Rolle zu spielen. Im Rahmen einer Metaanalyse ergaben sich bei BD Hinweise auf eine Beteiligung von drei in der Vergangenheit bereits mit SCZ assoziiert gefundenen CNVs: Duplikationen an den chromosomalen Loci 1q21.1 und 16p11.2 sowie Deletionen am Locus 3q29 [19]. Allerdings hielt nur der 16p11.2-Befund einer Korrektur für multiples Testen stand. Möglicherweise spielen CNVs auch nur bei BD-Patienten mit frühem Krankheitsbeginn eine signifikante Rolle [31, 43].

Bei MD fehlt bisher eine größere Metaanalyse zur möglichen Rolle von CNVs. In kleineren Studien gibt es zwar Hinweise auf einzelne CNVs, darunter auch Deletionen/Duplikationen am 16p11.2Locus, keiner dieser Befunde hält aber einer Korrektur für multiples Testen stand $[12,48]$.

Im Rahmen der wenigen, bei Patienten mit affektiven Störungen bisher durchgeführten exomweiten Sequenzierungen zur Identifizierung seltener Varianten konnten zwar erste Kandidatengene für BD und MD impliziert werden, genomweit signifikante Befunde liegen hier aber bisher nicht vor. Die implizierten Gene zeigen eine Anreicherung in der Cholesterolbiosynthese, den G-Protein-gekoppelten Rezeptoren sowie der Kalzium- und Kaliumsignalübertragung $[1,11,25]$. Zudem scheinen Gene angereichert zu sein, in denen erhöhte Raten von De-novo-Mutationen bei Patienten mit SCZ oder Autismus berichtet wurden [18]. Eine stichprobenmäßig sehr begrenzte Untersuchung von 79 ElternKind-Trios ergab erste Hinweise, dass

Nervenarzt 2019 · 90:99-106 https://doi.org/10.1007/s00115-018-0670-6

(c) Der/die Autor(en) 2019

\section{M. Nöthen · F. Degenhardt · A. J. Forstner}

\section{Durchbrüche im Verständnis der molekularen Ursachen psychiatrischer Störungen}

\section{Zusammenfassung}

Ein Beitrag genetischer Faktoren zur Entwicklung psychiatrischer Krankheiten wie den häufigen schizophrenen und affektiven Störungen war lange vermutet worden, deren zuverlässige molekulare Identifizierung gelingt aber erst seit wenigen Jahren. Möglich wurde dies durch technologische Fortschritte in der Charakterisierung der individuellen Ausprägungen des menschlichen Genoms und durch Zusammenführung großer genetischer Datensätze auf internationaler Ebene. Die Ergebnisse der genomweiten Analysen erlauben erstmals einen systematischen Einblick in krankheitsrelevante biologische Mechanismen, die integrierte Betrachtung unterschiedlicher Omiks-Ebenen trägt dabei wesentlich zur funktionellen Interpretation der genetischen Befunde bei. Die Ergebnisse der genetischen Untersuchungen zeigen auch, wie stark die
Ätiologien unterschiedlicher psychiatrischer Krankheiten überlappen, mit den größten Gemeinsamkeiten zwischen schizophrenen und bipolar affektiven Störungen. Das Ziel der Translation genetischer Befunde in die klinische Praxis wird auf unterschiedlichen Ebenen verfolgt, in der Regel sind aber aufwendige Folgestudien notwendig. Die diagnostische Untersuchung seltener genomischer Deletionen/Duplikationen (sog. Kopienzahlvarianten) bei Patienten mit Schizophrenie wird wahrscheinlich eines der ersten Beispiele klinischer Anwendung sein, hierfür werden derzeit die notwendigen Voraussetzungen definiert.

Schlüsselwörter

Affektive Störungen · Schizophrene Störungen - Genetik · Genomweite Assoziationsstudie $\cdot$ Kopienzahlvarianten

\section{Breakthrough in understanding the molecular causes of psychiatric disorders}

\section{Abstract}

A long-established hypothesis is that genetic factors contribute to the development of psychiatric diseases, including common illnesses such as schizophrenia and the affective disorders; however, reliable molecular identification of these factors represents a far more recent innovation. This has been rendered possible by technological advances in the individual characterization of the human genome and the combining of large genetic datasets at the international level. For the first time, the results of genome-wide analyses provide researchers with systematic insights into disease-relevant biological mechanisms. Here, the integrated analysis of different omics level data generates important insights into the functional interpretation of the genetic findings. The results of genetic studies also demonstrated the degree of etiological overlap between differing psychiatric disorders, with the greatest commonality having been observed to date between schizophrenia and bipolar affective disorder. Although the translation of genetic findings into routine clinical practice is being pursued at various levels, elaborate follow-up studies are typically necessary. The diagnostic investigation of rare genomic deletions/duplications (socalled copy number variants) in patients with schizophrenia is likely to represent one of the first examples of routine clinical application. The necessary prerequisites for this are currently being defined.

\section{Keywords}

Affective disorders · Schizophrenia · Genetics . Genome-wide association study · Copy number variant
De-novo-Mutationen auch zur Entstehung der BD beitragen könnten [24].

\section{Überlappende Ätiologien}

Schizophrene und affektive Störungen zeigen nicht nur auf der Ebene klinischer
Symptome weitreichende Überlappungen, die Erkrankungen treten auch gehäuft zusammen in Familien auf [30]. Es war also zu vermuten, dass ein Teil der genetischen Faktoren sich überschneidet, und durch Analyse der GWAS-Daten war es möglich, das Ausmaß der Überschnei- 
dung zu bestimmen. So konnte in der Analyse verschiedener psychiatrischer Erkrankungen gezeigt werden, dass die genetische Überlappung insbesondere zwischen schizophrenen und affektiven Störungen sehr groß ist (ca. $34 \%$ mit $\mathrm{MD}$ und $68 \%$ mit BD) und dass bestimmte SNPs diagnoseübergreifend zur Entstehung psychiatrischer Erkrankungen beitragen $[2,10,29]$. Die gemeinsamen Risikogene für SCZ und BD sind in bestimmten Stoffwechselwegen angereichert, z.B. in der synaptischen Langzeitpotenzierung oder in Glutamatsignalwegen [15]. Zudem konnten in einer großen Metaanalyse erstmals zwei genomweit signifikante Loci identifiziert werden, die sich zwischen Patienten mit SCZ und BD unterscheiden und damit offensichtlich zu krankheitsspezifischen Prozessen beitragen [5].

Unterstützt durch die genetischen Befunde werden psychiatrische Störungen zunehmend weniger kategorial, sondern eher mit dimensionalen Ansätzen konzeptualisiert [38]. In diese Richtung geht auch die vom National Institute of Mental Health eingeleitete Research-domaincriteria (RDoC)-Initiative, die die Pathophysiologie der Erkrankungen als zentrale Grundlage für neue Forschungs-, Diagnose- und Therapieansätze hervorhebt $[14,22]$.

\section{Ausblick}

Durch die Untersuchung immer gröBerer Patienten- und Kontrollkollektive wird die Identifizierung krankheitsassoziierter genomischer Regionen bei psychiatrischen Krankheiten weiter voranschreiten [56]. Der genomweite Ansatz dieser Untersuchungen ermöglicht eine systematische Kartierung der beteiligten biologischen Faktoren. Solange damit neue, bisher nicht implizierte biologische Zusammenhänge identifiziert werden, ist eine Erweiterung der genetischen Datensätze von großem Nutzen. Ein detailliertes mechanistisches Verständnis der biologischen Zusammenhänge verlangt allerdings zusätzliche Untersuchungen. Mittlerweile steht der Forschung dafür ein großes Methodenrepertoire zur Verfügung. Dieses schließt die Untersuchung biologischer Effekte humaner
Mutationen in anderen Spezies (z.B. CRISPR/Cas-induzierte Mutationen in Mausmodellen; [8]), die Generierung genetisch stratifizierter menschlicher Gehirnzellen und Organoide durch induzierte pluripotente Stammzellen [53] sowie die Untersuchung genetischer Effekte auf der Ebene intermediärer Phänotypen ein (z. B. Struktur und Funktion des Gehirns mithilfe bildgebender Verfahren, [6]).

Eine Herausforderung für funktionelle Untersuchungen stellt die Modellierung des polygenen Beitrags dar. Die auf den umfangreichen GWAS-Daten basierenden polygenen Risikoscores (PRS, z.B. [23]), mit denen die polygene Disposition auf der individuellen Ebene, sowohl bei Patienten als auch bei Gesunden, quantifiziert werden kann, werden hier als Durchbruch angesehen. Mithilfe der PRS können z. B. Untersuchungen intermediärer Phänotypen für den genetischen Beitrag stratifiziert werden [13]. Aber auch im experimentellen Modell gibt es erste erfolgreiche Ansätze zur funktionellen Analyse des polygenen Beitrags [20].

\section{》) Die funktionellen Konsequenzen der meisten GWAS-Befunde betreffen die Genregulation}

Die allermeisten GWAS-Befunde beipsychiatrischen Erkrankungen liegen in Bereichen des Genoms, die nicht direkt in Proteine umgesetzt werden. Die funktionellen Konsequenzen betreffen in diesem Fall höchstwahrscheinlich die Genregulation. Das krankheitsrelevante Gen muss aber nicht unbedingt das der assoziierten Variante nächstgelegene sein. Hilfreich sind dann Studien, die z.B. systematisch den Einfluss genetischer Varianten auf die Expression von Genen untersuchen (sog. „expression quantitative trait loci“, eQTLs). Da die Regulation der Genexpression häufig gewebebzw. zellspezifisch erfolgt, benötigt man bei psychiatrischen Erkrankungen diese Daten für relevante Gewebe bzw. Zellen des Gehirns [50]. Mittlerweile gibt es international koordinierte Bemühun- gen, solche Daten systematisch für alle Gewebe/Zelltypen beim Menschen zugenerieren (u.a. Genotype-Tissue Expression [GTEx] project; CommonMind Consortium). Wenn man allerdings bedenkt, dass die genetische Variante möglicherweise in einer DNA-Sequenz liegt, die nur zur Regulation des Gens im Rahmen des Krankheitsprozesses verwendet wird, und damit der funktionelle Effekt im Normalzustand gar nicht sichtbar ist, kann man sich vorstellen, vor welchen methodischen Herausforderungen die Generierung solcher Datensätze speziell für psychiatrische Erkrankungen steht.

Die Entwicklung neuer Medikamente zur Therapie psychiatrischer Erkrankungen stagniert seit mehreren Jahren, mehrere große Pharmaunternehmen haben sich aus dem Indikationsgebiet zurückgezogen [21]. Von der genetisch basierten Charakterisierung biologischer Stoffwechselwege erhofft man sich hier ein neues Momentum [7]. Die Tatsache, dass in den genetischen Studien Gene identifiziert werden, die für etablierte „drug targets“ (z. B. Dopamin-D2-Rezeptor bei der SCZ) bzw. für deren nahe Interaktionspartner kodieren, dient dabei als "proof of principle“. Auf Basis der genetischen Befunde sind u. a. für die SCZ das „synaptic pruning“, die glutamaterge Neurotransmission oder bei der BD Prozesse der Kalziumsignalwege als vielversprechende biologische Interventionsfelder identifiziert worden [36, 42, 49, 51].

\section{》) Voraussetzungen zur Translation genetischer Befunde in die Diagnostik werden derzeit eruiert}

Während die Entwicklung neuer Medikamente ein langwieriger und höchst aufwendiger Prozess ist, kann die Translation genetischer Befunde in die diagnostische Praxis im Prinzip sehr viel rascher erfolgen. So ist eine genomweite Untersuchung auf CNVs heutzutage diagnostische Routine bei Patienten mit Intelligenzminderung oder einer Störung aus dem Autismusspektrumformenkreis. Obwohl es auch Vorstöße für eine Anwendung bei der SCZ gibt [3], 
ist insbesondere der klinische Nutzen noch nicht überzeugend dargestellt. Der klinische Nutzen könnte z.B. darin liegen, dass Trägern bestimmter CNVs ein dem psychischen und somatischen Symptomspektrum angepasstes klinisches Management empfohlen wird [60]. Da CNVs vor allem bei schizophrenen Patienten mit zusätzlichen Symptomen bzw. Auffälligkeiten beobachtet werden, stellt sich auch die Frage nach den spezifischen Indikationskriterien für eine CNV-Untersuchung.

Wie verhält es sich mit den PRS in der Diagnostik? Der erste Einsatz der PRS in der Diagnostik könnte gekoppelt an die CNV-Diagnostik erfolgen, da der polygene Hintergrund die Penetranz der CNVs beeinflussen und bei einer prädiktiven (vorhersagenden) Diagnostik bei Verwandten erkrankter CNV-Träger das Risiko modifizieren kann [57]. Weitere diagnostische Einsatzgebiete für den PRS sind analog zu anderen multifaktoriellen Krankheiten vorstellbar [59], wie z. B. im Kontext einer Therapieentscheidung, bei der Definition von Hochrisikogruppen für präventive Interventionen oder im Rahmen der individuellen Lebensplanung (u. a. $[16,33]$ ). Für alle diese Anwendungsbereiche muss aber zunächst der Nutzen in wissenschaftlichen Studien gezeigt werden. Eine weitere Anwendungsperspektive ist die Abgrenzung von homogeneren Subgruppen von Patienten und darauf aufbauend die Etablierung ätiologisch basierter Klassifizierungsschemata $[5,22,38]$.

Bei psychiatrischen Erkrankungen entfaltet sich der genetische Beitrag nur im Zusammenspiel mit äußeren Einflussfaktoren. Mit zunehmender Identifizierung der genetischen Faktoren ergeben sich neue Möglichkeiten der Untersuchung von Gen-Umwelt-Interaktionen [58]. In epidemiologischen Studien eröffnet die Einbeziehung genetischer Daten erstmals die Möglichkeit, den Einfluss äußerer Risikofaktoren auf Kausalität zu testen (Stichwort „Mendelian randomization“, z. B. [27]). Auf der molekularen Seite gelingt es zunehmend, in relevanten Geweben/Zellen biologische Signaturen äußerer Einflüsse zu identifizieren, z.B. durch die Untersuchung von DNA-Methylierung (für eine
Übersicht siehe z.B. [26]). Technologische Fortschritte (z.B. MethylierungsMikroarrays) erlauben mittlerweile auch hier genomweite Untersuchungen. Dadurch gewinnt das Forschungsgebiet der Epigenetik bei psychiatrischen Erkrankungen ein zusätzliches Momentum.

Nicht zuletzt haben die genetischen Untersuchungen entscheidend dazu beigetragen, psychiatrische Erkrankungen als Erkrankungen komplexer biologischer Netzwerke zu verstehen. Diese Netzwerke im Sinne eines systemmedizinischen Ansatzes umfassender zu verstehen, ist die große Herausforderung für die Zukunft.

\section{Fazit für die Praxis}

- Der genetische Beitrag zu affektiven und schizophrenen Störungen ist bei der Mehrzahl der Patienten durch ein Zusammenspiel vieler, in bestimmten biologischen Stoffwechselwegen angereicherter Gene bedingt (polygener Beitrag).

- Eine Subgruppe von Patienten mit schizophrenen Störungen weist spezifische genomische Deletionen/ Duplikationen auf (sog. CNVs), mit einem jeweils größeren Beitrag zur Entwicklung der Erkrankung.

- Die Identifizierung kleinerer Mutationen mit großen Beiträgen steht erst am Anfang, dazu werden in naher Zukunft viele Patienten in wissenschaftlichen Studien großflächig sequenziert.

- Die systematische Aufklärung der biologischen Ursachen durch genetische und nachfolgende funktionelle Untersuchungen wird Ausgangspunkt für die Entwicklung neuer medikamentöser Strategien sein.

- Für die Umsetzung genetischer Befunde in neue diagnostische Möglichkeiten (z. B. CNV-Diagnostik) werden derzeit die Rahmenbedingungen erarbeitet (z. B. Indikationskriterien).

\section{Korrespondenzadresse}

\section{Markus M. Nöthen}

Institut für Humangenetik, Universitätsklinikum Bonn

Sigmund-Freud-Str. 25, 53127 Bonn,

Deutschland

markus.noethen@uni-bonn.de

\section{Einhaltung ethischer Richtlinien}

Interessenkonflikt. M.M. Nöthen, F. Degenhardt und A.J. Forstner geben an, dass kein Interessenkonflikt besteht.

Dieser Beitrag beinhaltet keine von den Autoren durchgeführten Studien an Menschen oder Tieren.

Open Access. Dieser Artikel wird unter der Creative Commons Namensnennung 4.0 International Lizenz (http://creativecommons.org/licenses/by/4.0/deed. de) veröffentlicht, welche die Nutzung, Vervielfältigung, Bearbeitung, Verbreitung und Wiedergabe in jeglichem Medium und Format erlaubt, sofern Sie den/die ursprünglichen Autor(en) und die Quelle ordnungsgemäßnennen, einen Link zur Creative Commons Lizenz beifügen und angeben, ob Änderungen vorgenommen wurden.

\section{Literatur}

1. Amin N, Jovanova O, Adams $\mathrm{HH}$ et al (2017) Exome-sequencing in a large population-based study reveals a rare Asn396Ser variant in the LIPG gene associated with depressive symptoms. Mol Psychiatry 22:537-543

2. Anttila V, Bulik-Sullivan B, Finucane HK et al (2018) Analysis of shared heritability in common disorders of the brain. Science eaap8757:360. https://doi. org/10.1126/science.aap8757

3. Baker K, Costain G, Fung WL et al (2014) Chromosomal microarray analysis - a routine clinical genetictestfor patients with schizophrenia. Lancet Psychiatry 1:329-331

4. Bienvenu OJ, Davydow DS, Kendler KS (2011) Psychiatric 'diseases' versus behavioral disorders and degree of genetic influence. Psychol Med 41:33-40

5. Bipolar Disorder and Schizophrenia Working Group of the Psychiatric Genomics Consortium (2018) Genomic dissection of bipolar disorder and schizophrenia, including 28 subphenotypes. Cell 173:1705-1715.e16

6. Bogdan R, Salmeron BJ, Carey CE et al (2017) Imaging genetics and genomics in psychiatry: a critical review of progress and potential. Biol Psychiatry 82:165-175

7. Breen G, Li Q, Roth BL et al (2016) Translating genome-wide association findings into new therapeutics for psychiatry. Nat Neurosci 19:1392-1396

8. CaligiuriSP, KennyPJ(2018)Thepromise of genome editing for modeling psychiatric disorders. Neuropsychopharmacology 43:223-224

9. Cichon S, Mühleisen TW, DegenhardtFA etal (2011) Genome-wide association study identifies genetic variation in neurocan as a susceptibility factor for bipolar disorder. Am JHum Genet 88:372-381 
Hier steht eine Anzeige.

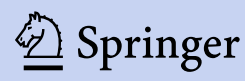


10. Cross-Disorder Group of the Psychiatric Genomics Consortium (2013) Identification of risk loci with shared effects on five major psychiatric disorders: a genome-wide analysis. Lancet 381:1371-1379

11. Cruceanu C, Schmouth JF, Torres-Platas SG et al (2017) Rare susceptibility variants for bipolar disorder suggest a role for $\mathrm{G}$ protein-coupled receptors. Mol Psychiatry. https://doi.org/10. 1038/mp.2017.223

12. Degenhardt F, Priebe $L$, Herms $S$ et al (2012) Association between copy number variants in 16p11.2 and major depressive disorder in a German case-control sample. Am J Med Genet B Neuropsychiatr Genet 159B:263-273

13. Dima D, Breen G (2015) Polygenic risk scores in imaging genetics: Usefulness and applications. JPsychopharmacol 29:867-871

14. Doherty JL, Owen MJ (2014) The research domain criteria: moving the goalposts to change the game. Br JPsychiatry 204:171-173

15. Forstner AJ, Hecker J, Hofmann A et al (2017) Identification of shared risk loci and pathways for bipolar disorder and schizophrenia. PLoS ONE 12:e171595

16. Frank J, Lang M, Witt SH et al (2015) Identification of increased genetic risk scores for schizophrenia in treatment-resistant patients. Mol Psychiatry 20:150-151

17. Fromer $\mathrm{M}$, Pocklington $\mathrm{AJ}$, Kavanagh $\mathrm{DH}$ et al (2014) De novo mutations in schizophrenia implicate synaptic networks. Nature 506:179-184

18. Goes FS, Pirooznia M, Parla JS et al (2016) Exome sequencing of familial bipolar disorder. JAMA Psychiatry 73:590-597

19. Green EK, Rees E, Walters JT et al (2016) Copy number variation in bipolar disorder. Mol Psychiatry 21:89-93

20. Hoekstra SD, Stringer S, Heine VM et al (2017) Genetically-informed patient selection for iPSC studies of complex diseases May aid in reducing cellular heterogeneity. Front Cell Neurosci 11:164

21. Hyman SE (2013) Psychiatric drug development: diagnosing a crisis. Cerebrum 2013:5

22. Insel T, Cuthbert B, Garvey M et al (2010) Research domain criteria $(\mathrm{RDoC})$ : toward a new classification framework for research on mental disorders. Am J Psychiatry 167:748-751

23. International Schizophrenia Consortium, Purcell SM, Wray NR et al (2009) Common polygenic variation contributes to risk of schizophrenia and bipolar disorder. Nature 460:748-752

24. Kataoka M, Matoba N, Sawada T et al (2016) Exome sequencing for bipolar disorder points to roles of de novo loss-of-function and protein-altering mutations. Mol Psychiatry 21:885-893

25. Kato T (2015) Whole genome/exome sequencing in mood and psychotic disorders. Psychiatry Clin Neurosci 69:65-76

26. Klengel T, Binder EB (2015) Epigenetics of stress-related psychiatric disorders and gene $\times$ environment interactions. Neuron 86:1343-1357

27. Köhler CA, Evangelou E, Stubbs B et al (2018) Mapping risk factors for depression across the lifespan: an umbrella review of evidence from meta-analyses and mendelian randomization studies. J Psychiatr Res 103:189-207

28. Lander ES, Linton LM, Birren B et al (2001) Initial sequencing and analysis of the human genome. Nature 409:860-921

29. Lee SH, Ripke S, Neale BM et al (2013) Genetic relationship between five psychiatric disorders estimated from genome-wide SNPs. Nat Genet 45:984-994
30. Lichtenstein P, Yip BH, Björk C et al (2009) Common genetic determinants of schizophrenia and bipolar disorder in Swedish families: a population-based study. Lancet 373:234-239

31. Malhotra D, McCarthy S, Michaelson JJ et al (2011) High frequencies of de novo CNVs in bipolar disorder and schizophrenia. Neuron 72:951-963

32. Marshall CR, Howrigan DP, Merico D et al (2017) Contribution of copy number variants to schizophrenia from a genome-wide study of 41,321 subjects. Nat Genet 49:27-35

33. Meier SM, Agerbo E, Maier R et al (2016) High loading of polygenic risk in cases with chronic schizophrenia. Mol Psychiatry 21:969-974

34. Mühleisen TW, Leber M, Schulze TG et al (2014) Genome-wide association study reveals two new risk loci for bipolar disorder. Nat Commun 5:3339

35. Mullins N, Lewis CM (2017) Genetics of depression: progress at last. Curr Psychiatry Rep 19:43

36. Neale BM, Sklar P (2015) Genetic analysis of schizophrenia and bipolar disorder reveals polygenicity but also suggests new directions for molecular interrogation. Curr Opin Neurobiol 30:131-138

37. Network and Pathway Analysis Subgroup of Psychiatric Genomics Consortium (2015) Psychiatric genome-wide association study analyses implicate neuronal, immune and histone pathways. Nat Neurosci 18:199-209

38. Owen MJ (2014) New approaches to psychiatric diagnostic classification. Neuron 84:564-571

39. Pardiñas AF, Holmans $P$, Pocklington $A J$ et al (2018) Common schizophrenia alleles are enriched in mutation-intolerant genes and in regions under strong background selection. Nat Genet 50:381-389

40. Pettersson E, Lichtenstein P, Larsson $\mathrm{H}$ et al (2018) Genetic influences on eight psychiatric disorders based on family data of 4408646 full and halfsiblings, and genetic data of 333748 cases and controls. Psychol Med. https://doi.org/10.1017/ S0033291718002039

41. Power RA, Kyaga S, Uher R et al (2013) Fecundity of patients with schizophrenia, autism, bipolar disorder, depression, anorexia nervosa, or substance abuse vs their unaffected siblings. JAMA Psychiatry 70:22-30

42. Presumey J, Bialas AR, Carroll MC (2017) Complement system in neural synapse elimination in development and disease. Adv Immunol 135:53-79

43. Priebe L, Degenhardt FA, Herms S et al (2012) Genome-wide survey implicates the influence of copy number variants (CNVs) in the development of early-onset bipolar disorder. Mol Psychiatry $17: 421-432$

44. Propping P (1989) Psychiatrische Genetik - Befunde und Konzepte. Springer, Berlin, Heidelberg

45. Psychiatric GWAS Consortium Bipolar Disorder Working Group (2011) Large-scale genome-wide association analysis of bipolar disorder identifies a new susceptibility locus near ODZ4. Nat Genet 43:977-983

46. Purcell SM, Moran JL, Fromer M et al (2014) A polygenic burden of rare disruptive mutations in schizophrenia. Nature 506:185-190

47. Rietschel M, Mattheisen M, Frank J et al (2010) Genome-wide association-, replication-, and neuroimaging study implicates HOMER1 in the etiology of major depression. Biol Psychiatry 68:578-585

48. Rucker JJ, Tansey KE, Rivera M et al (2016) Phenotypic association analyses with copy number variation in recurrent depressive disorder. Biol Psychiatry 79:329-336

49. Schizophrenia Working Group of the Psychiatric Genomics Consortium (2014) Biological insights from 108 schizophrenia-associated genetic loci. Nature 511:421-427

50. Schulz H, Ruppert AK, Herms S et al (2017) Genome-wide mapping of genetic determinants influencing DNA methylation and gene expression in human hippocampus. Nat Commun 8:1511

51. Sekar A, Bialas AR, de Rivera $H$ et al (2016) Schizophrenia risk from complex variation of complement component 4. Nature 530:177-183

52. Singh T, Kurki MI, Curtis D et al (2016) Rare loss-offunction variants in SETD1A are associated with schizophrenia and developmental disorders. Nat Neurosci 19:571-577

53. Soliman MA, Aboharb F, Zeltner N et al (2017) Pluripotent stem cells in neuropsychiatric disorders. Mol Psychiatry 22:1241-1249

54. Stahl E, Breen G, Forstner A et al (2018) Genomewide association study identifies 30 loci associated with bipolar disorder. bioRxiv 173062. https://doi. org/10.1101/173062

55. Sullivan PF (2010) The psychiatric GWAS consortium: big science comes to psychiatry. Neuron 68:182-186

56. Sullivan PF, Agrawal A, Bulik CM et al (2018) Psychiatric genomics: an update and an agenda. Am J Psychiatry 175:15-27

57. Tansey KE, Rees E, Linden DE et al (2016) Common alleles contribute to schizophrenia in CNV carriers. Mol Psychiatry 21:1085-1089

58. Thomas D (2010) Gene-environment-wide association studies: emerging approaches. Nat Rev Genet 11:259-272

59. Torkamani A, Wineinger NE, Topol EJ (2018) The personal and clinical utility of polygenic risk scores NatRev Genet 19:581-590

60. Wolfe K, Strydom A, Morrogh D et al (2016) Chromosomal microarray testing in adults with intellectual disability presenting with comorbid psychiatric disorders. Eur J Hum Genet 25:66-72

61. Wray NR, Ripke S, Mattheisen M et al (2018) Genome-wide association analyses identify 44 risk variants and refine the genetic architecture of major depression. Nat Genet 50:668-681 